\title{
A NOTE ON THE PAPER "A SURVEY OF THE WORK OF GEORGE LUSZTIG" BY R. CARTER
}

\author{
EDITORIAL COMMITTEE
}

In Section 16 of the paper "A survey of the work of George Lusztig" ([C]) appeared in the previous volume in Nagoya Math. Journal, R. Carter discusses the canonical bases of quantized enveloping algebras constructed by G. Lusztig. He briefly mentions a connection with the work of M. Kashiwara. In this note, we want to make a correction and add some remarks to make this connection more precise.

Correction. The sentence

"This basis was also independently proved to exist by Kashiwara by using a different method,"

in Carter's paper, page 32, line 12 should be replaced by

"This basis was also subsequently proved to exist by Kashiwara by using a different method,".

In preparing his article, Carter had to leave the managing editor to edit his draft at the last step. Carter's original draft was written in the latter form. The managing editor changed it to the former following the suggestion of the referee. However, the editorial committee realizes that the present expression is not correct, so decides to put this sentence back to the original form, and add more explanation concerning the relation to Kashiwara's work.

We follow the notation in [C]. In particular, $U=U_{v}(\mathfrak{g})$ is the quantized enveloping algebra associated to $\mathfrak{g}, U^{-}$is the lower triangular subalgebra of $U$, and $L$ is a certain $\mathbb{C}\left[v^{-1}\right]$-lattice of $U^{-}$. In [L1] Lusztig constructed in the case where $\mathfrak{g}$ is of type $A, D, E$, the canonical basis of $U^{-}$. To do so he needed to first construct a "basis at $v=\infty$ " of $U^{-}$that is a basis of $L / v^{-1} L$. From these he constructed for a finite dimensional $U$-module $M$, a canonical basis of $M$ and a basis of $M$ at $v=\infty$, that is a basis of $L_{M} / v^{-1} L_{M}$ for a certain $\mathbb{C}\left[v^{-1}\right]$-lattice $L_{M}$ of $M$. Independently, in [K1], Kashiwara constructed in the case where $\mathfrak{g}$ is of type $A, B, C, D$ a basis of 
$M$ at $v=0$, that is a basis of $\bar{L}_{M} / v \bar{L}_{M}$ where $\bar{L}_{M}$ is a certain $\mathbb{C}[v]$-lattice of $M$; he called it the "crystal basis" of $M$. The fact that $\bar{L}_{M}$ coincides with the image of $L_{M}$ under the bar operation (when both are defined) was proved in [L2].

In [K2], Kashiwara constructed a basis at $v=0$ of $U^{-}$for any type of $\mathfrak{g}$ that he calls the "crystal basis"; moreover, under some influence of [L1], he constructed a basis of $U^{-}$that he calls the "global crystal basis". (His construction is applicable in the more general symmetrizable Kac-Moody case.) In [L2] it was shown that for type $A, D, E$, Kashiwara's global crystal basis coincides with Lusztig's canonical basis.

In [L3] Lusztig extended the construction of the canonical basis of $U^{-}$ given in [L1] to the symmetric Kac-Moody case, using the theory of perverse sheaves. In [GL] it was shown that this agrees with Kashiwara's construction $[\mathrm{K} 2]$.

\section{REFERENCES}

[C] R. Carter, A survey of the work of George Lusztig, Nagoya Math. J., 182 (2006), $1-45$.

[GL] I. Grojnowski and G. Lusztig, A comparison of bases of quantized enveloping algebras, Linear algebaric groups and their representations, Contemp. Math. 153 (1993), pp. 11-19.

[K1] M. Kashiwara, Crystalizing the q-analogue of universal enveloping algebras, Comm. Math. Phys., 132 (1990), 249-260.

[K2] M. Kashiwara, On crystal bases of the q-analogue of universal enveloping algebras, Duke Math. J., 63 (1991), 465-516.

[L1] G. Lusztig, Canonical bases arising from quantized enveloping algebras, J. Amer. Math. Soc., 3 (1990), 447-498.

[L2] G. Lusztig, Canonical bases arising from quantized enveloping algebras II, Progr. Theor. Phys. Suppl., 102 (1990), 175-201.

[L3] G. Lusztig, Quivers, perverse sheaves and quantized enveloping algebras, J. Amer. Math. Soc., 4 (1991), 365-421. 